\title{
Lewy body pathology is more prevalent in older individuals with mitochondrial disease than controls
}

\author{
Daniel Erskine ${ }^{1,2}$ (1) $\cdot$ Amy K. Reeve ${ }^{1,2} \cdot$ Tuomo Polvikoski $^{1,2} \cdot$ Andrew M. Schaefer $^{1,3} \cdot$ Robert W. Taylor $^{1,3}$. \\ Nichola Z. Lax ${ }^{1,2} \cdot$ Omar El-Agnaf $^{4} \cdot$ Johannes Attems ${ }^{2} \cdot$ Gráinne S. Gorman $^{1,2,3} \cdot$ Doug M. Turnbull ${ }^{1,2,3} \cdot$ Yi Shau Ng ${ }^{1,2,3}$
}

Received: 31 October 2019 / Revised: 22 November 2019 / Accepted: 22 November 2019 / Published online: 28 November 2019

(c) The Author(s) 2019

Mitochondrial diseases arise due to defects in mitochondrial DNA (mtDNA) or nuclear mitochondrial genes (nDNA), leading to impaired mitochondrial oxidative phosphorylation and dysfunction of organs with particularly high energy requirements. Primary mitochondrial diseases affect 1 in 4300 individuals in the UK, making them amongst the most common heritable neurological conditions [2]. Mitochondrial dysfunction has also been linked to deposition of several sporadic age-associated pathologies, including neurofibrillary tangles of hyperphosphorylated tau protein [3] and Lewy body (LB) pathology consisting of aggregated $\alpha$-synuclein [4], pathological features of Alzheimer's disease (AD) and Parkinson's disease (PD)/dementia with Lewy bodies (DLB), respectively. Therefore, we sought to determine whether older individuals with mitochondrial diseases were at increased risk of developing age-associated neurodegenerative pathologies using post-mortem brain samples collected prospectively at Newcastle Brain Tissue Resource (NBTR).

We identified 17 cases with clinical and molecular confirmation of mitochondrial disease and sufficient tissue

Electronic supplementary material The online version of this article (https://doi.org/10.1007/s00401-019-02105-w) contains supplementary material, which is available to authorized users.

Daniel Erskine

daniel.erskine@ncl.ac.uk

1 Wellcome Centre for Mitochondrial Research, Newcastle University, Newcastle upon Tyne N2 4HH, UK

2 Translational and Clinical Research Institute, Newcastle University, Newcastle, UK

3 NHS Highly Specialised Service for Rare Mitochondrial Disorders, Newcastle upon Tyne Hospitals NHS Foundation Trust, Newcastle upon Tyne, UK

4 Neurological Disorders Research Centre, Qatar Biomedical Research Institute, Hamad Bin Khalifa University, P.O. Box 34110, Doha, Qatar collected at NBTR between 2004 and 2019 (m.3243A $>\mathrm{G}$ $N=6 ;$ m.8344A $>$ G $N=1 ; P O L G N=5 ; S D H A N=1$; RRM $2 B N=1$; multiple mtDNA deletions $N=2$, single large-scale mtDNA deletion $N=1$; Supplementary Table 1). Each case was stained with antibodies against hyperphosphorylated tau, amyloid- $\beta$ and $\alpha$-synuclein (Supplementary Data 1), and pathological stage was determined using international consensus guidelines [8]. We found that 5/17 mitochondrial cases $(29.4 \%)$ had LB pathology, whilst a case without LB had pathology consistent with progressive supranuclear palsy (PSP; case 7). In all cases, LB pathology was labelled by antibodies against fibrillar $\alpha$-synuclein and $\alpha$-synuclein phosphorylated at serine 129 , as in idiopathic LB disease (Fig. 1). LB pathology occurred in 4/9 (44.4\%) cases with nDNA mutations, whilst it was present only in 1 case of mtDNA pathogenic variant $(1 / 8 ; 12.5 \%)$. In contrast to $L B$ pathology, amyloid- $\beta$ and tau pathology were typical of the level observed in neurologically normal elderly brains and we found no evidence of age-associated pathologies such as TDP-43, age-related tau astrogliopathy and perivascular neuritic dystrophy (Supplementary Table 1). However, one case (case 11) had focal neocortical tau pathology, despite having no significant pathology in the entorhinal cortex or hippocampus (Supplementary Figure 1).

We compared the proportion of mitochondrial cases with LB pathology to that reported in all neurologically normal control cases aged over 50 collected at NBTR since 2010, the time at which all three pathologies were systematically evaluated using the aforementioned neuropathological guidelines in control cases $(N=82)$. LB pathology was less prevalent amongst control cases $(9 / 82,10.9 \%)$ than mitochondrial disease cases $\left(5 / 17,29.4 \% ; \chi^{2}=3.94, p=0.047\right)$, despite mitochondrial cases being significantly younger ( $63 \pm 10$ years) than controls ( $83 \pm 12$ years; $p<0.0001)$. The proportion of control cases with incidental LB pathology was similar to another report of LB prevalence in control cases over 60 years old [1]. 


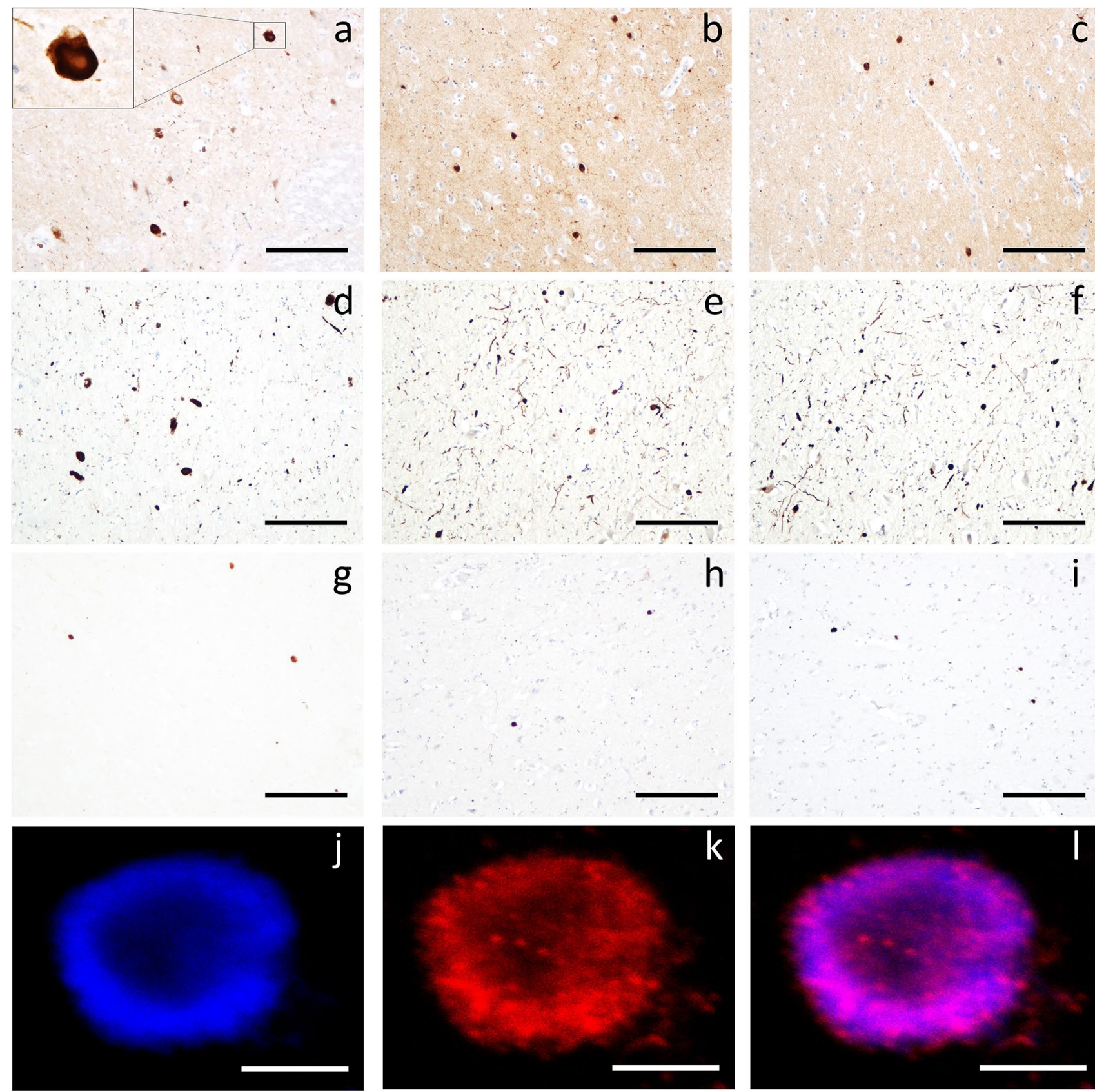

Fig. 1 Lewy body pathology in mitochondrial disease cases. Representative examples of LB pathology in the substantia nigra (a), amygdala (b) and inferotemporal cortex (c) of case 2; LB pathology in the nucleus of Meynert of case 6 stained with KM51 (d), Syn-F2 (e)

Despite the small size of this cohort, the present findings suggest LB pathology is more prevalent amongst older individuals with mitochondrial disease, particularly those with nDNA mutations, than in a comparable control population. Familial PD resulting from mitochondrial nDNA mutations does not always result in LB pathology, and the prevalence of LB pathology in Parkin cases is 33\% based on reports from 18 cases, a comparable figure in a similarly sized and pS129 (f); LB pathology in the amygdala of case 8 stained with KM51 (g), Syn-F2 (h) and pS129 (i); an LB in the substantia nigra of case 14 labelled with Syn-F2 (j), pS129 (k) and merged (l). Scale bars $100 \mu \mathrm{m}(\mathbf{a}-\mathbf{i}), 5 \mu \mathrm{m}(\mathbf{j}-\mathbf{l})$

cohort to our presently reported findings [6]. As mitochondrial dysfunction is associated with phenotypes of ageing [7], one could speculate that increased levels of LB pathology in this population reflect an acceleration of ageing; however, as we did not find increased levels of other ageassociated pathologies, it seems unlikely that accelerated ageing alone could explain these findings. Nevertheless, it is difficult to make strong conclusions based on such a small 
cohort, but we hope these findings will stimulate further study of LB pathology in this population.

The clinical significance of LB pathology in this population is unclear as mitochondrial disease alone can cause significant neurodegeneration leading to parkinsonism and cognitive impairment [5] (Supplementary Data 2 and 3). We suggest the present findings highlight the need for further study of the prevalence of LB pathology in older mitochondrial disease patients. If confirmed in a larger cohort, the present findings suggest mitochondrial dysfunction and an elevated (though not absolute) risk of developing LB pathology is a feature of both aged mitochondrial disease cases and some forms of familial PD characterised by mutations in mitochondrial proteins such as Parkin, raising questions about the distinction between these disorders. Further understanding of LB pathology in this population is important to understand its clinical relevance in the sub-set of mitochondrial diseases cases affected by it, and to determine the role of mitochondrial dysfunction in the genesis of LB pathology.

Acknowledgements This study was funded by the Wellcome Centre for Mitochondrial Research at Newcastle University (203105/Z/16/Z). D.E. is funded by an Alzheimer's Research UK Fellowship (ARUKRF2018C-005). Y.S.N. holds a NIHR funded academic clinical lectureship (CL-2016-01-003). Brain tissue was collected by Newcastle Brain Tissue Resource, which is funded in part by a Grant from the UK Medical Research Council (G0400074), by NIHR Newcastle Biomedical Research Centre awarded to the Newcastle upon Tyne NHS Foundation Trust and Newcastle University, and by a Grant from the Alzheimer's Society and Alzheimer's Research UK as part of the Brains for Dementia Research Project. The authors would like to thank Debbie Lett from Newcastle Brain Tissue Resource for her assistance with case identification, and Newcastle University Bioimaging Core Facility for assistance with confocal microscopy. The tissue for case 7 was provided with assistance from the Edinburgh Brain Bank and the authors would like to express their thanks to Professor Malcolm MacLeod and Professor Colin Smith. Molecular diagnoses were provided by the NHS "Rare Mitochondrial Disorders of Adults and Children Diagnostic Service" in Newcastle upon Tyne (https://www.newcastle-mitochondr ia.com/), with funding from UK NHS Specialist Commissioners. The views expressed are those of the authors and not necessarily those of the NHS, the NIHR or the UK Department of Health.
Open Access This article is distributed under the terms of the Creative Commons Attribution 4.0 International License (http://creativeco mmons.org/licenses/by/4.0/), which permits unrestricted use, distribution, and reproduction in any medium, provided you give appropriate credit to the original author(s) and the source, provide a link to the Creative Commons license, and indicate if changes were made.

\section{References}

1. Frigerio R, Fujishiro H, Ahn TB, Josephs KA, Maraganore DM, DelleDonne A et al (2011) Incidental Lewy body disease: do some cases represent a preclinical stage of dementia with Lewy bodies? Neurobiol Aging 32:857-863. https://doi.org/10.1016/j.neurobiola ging.2009.05.019

2. Gorman GS, Schaefer AM, Ng Y, Gomez N, Blakely EL, Alston CL et al (2015) Prevalence of nuclear and mitochondrial DNA mutations related to adult mitochondrial disease. Ann Neurol 77:753-759. https://doi.org/10.1002/ana.24362

3. Melov S, Adlard PA, Morten K, Johnson F, Golden TR, Hinerfeld D et al (2007) Mitochondrial oxidative stress causes hyperphosphorylation of tau. PLoS ONE 2:e536. https://doi.org/10.1371/ journal.pone.0000536

4. Mullin S, Schapira A (2013) alpha-Synuclein and mitochondrial dysfunction in Parkinson's disease. Mol Neurobiol 47:587-597. https://doi.org/10.1007/s12035-013-8394-x

5. Ng YS, Lax NZ, Bindoff LA, Turnbull DM (2019) Mitochondrial neurodegenerative disorders I: parkinsonism and cognitive deficits. Diagnosis and management of mitochondrial disorders. Springer, Cham, pp 223-239

6. Schneider SA, Alcalay RN (2017) Neuropathology of genetic synucleinopathies with parkinsonism: review of the literature. Mov Disord 32:1504-1523. https://doi.org/10.1002/mds.27193

7. Trifunovic A, Larsson NG (2008) Mitochondrial dysfunction as a cause of ageing. J Intern Med 263:167-178. https://doi.org/10. 1111/j.1365-2796.2007.01905.x

8. Walker L, McAleese KE, Erskine D, Attems J (2019) Neurodegenerative diseases and ageing. Biochemistry and cell biology of ageing: part II clinical science. Springer, Singapore, pp 75-106

Publisher's Note Springer Nature remains neutral with regard to jurisdictional claims in published maps and institutional affiliations. 